\title{
2D Modelling of Mechanical Stress Evolution and Electromigration in Confined Aluminum Interconnects
}

\author{
V. Petrescu, A. J. Mouthaan
}

\begin{abstract}
A complete description for mechanical stress evolution and electromigration in confined $\mathrm{Al}$ interconnects, taking into account the microstructure features, is presented in this paper. In the last years there were proposed several $1 D$ models for the time-dependent evolution of the mechanical stress $[1,2,3]$ in $\mathrm{Al}$ interconnect lines, since the time to failure of the line can be related to the time a critical value of the stress is reached [4]. The present paper extends and improves the existing models in 2D using a two dimensional simulator based on finite element method. Also, the model makes an attempt to relate the stress/ vacancy concentration evolution with the early resistance change of the Al line [5].
\end{abstract}

\section{Introduction}

Metallization failure in integrated circuits is a problem in the semiconductor electronics industry ever since the advent of thin film technology. A serious reliability concern in the last decade for IC manufacturers is electromigration, defined as mass transport resulting from the momentum transfer between the conducting electrons and lattice atoms. The aluminum interconnect line is considered confined by the silicon substrate and a rigid passivation layer. Aluminum thin films are characterised by small grains which offers many high diffusivity grainboundaries paths, making math transport easier along these paths. This mass transport can lead to void growth (at places where a depletion of atoms occurs) and to hillock formation and passivation cracking at places where

V. Petrescu and A.J. Mouthaan are with the MESA Research Institute, University of Twente, Dept. of Electrical Engineering, P.O. Box 217, 7500 AE Enschede, The Netherlands, E-mail: v.petrescu@el.utwente.nl. a high concentration of atoms exist. This depletion/ accumulation of atoms will generate a mechanical stress, which if it grows large enough, can cause line failure. So that the time to failure of the metallic line can be determined by the time required to reach a critical, or failure, stress. The value of critical stress depends on passivation type, thickness. An other technique that could predict the time to failure of the metal line is the measurement of the small resistance changes in the early stages of electromigration. Sensitive measurements of the resistance of aluminum based metalisation stripes that have been electrically stressed with large current densities, show a linear increase in time.

\section{Model description}

The atomic flow due to electromigration is modeled using a vacancy mechanism. In the model both electromigration and stressmigration are described in terms of the evolution of vacancy concentration. Consider a near-bamboo interconnect line (the line consists of a successive alternation of polycrystalline regions or clusters and single crystal regions as indicated in fig. 1), in the model the material flow is due to diffusion of atoms/vacancies along grain boundaries. Places where clusters terminate at bamboo grains are flux divergent sites, and also sites with the maximum value of mechanical stress. In the model is assumed that there is a linear relation between a change in vacancy concentration and small resistance changes. 


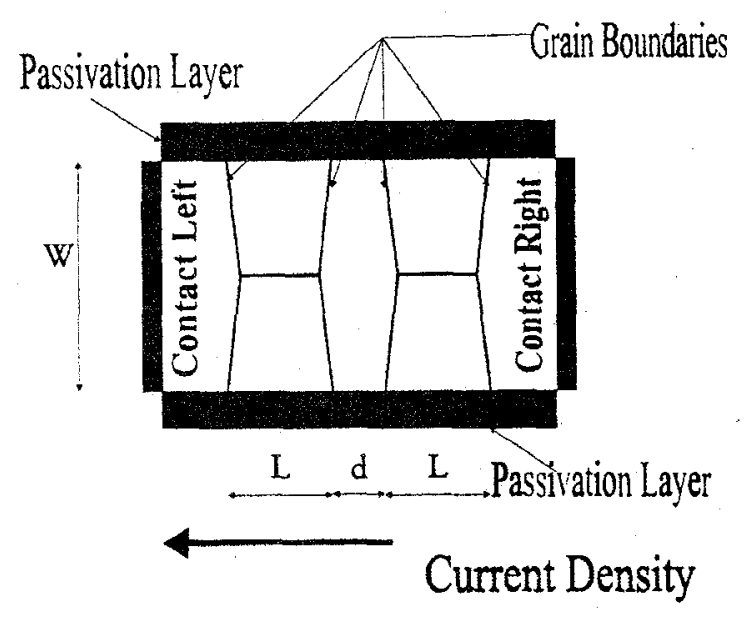

Fig. 1. Near-bamboo interconnect line

\section{Equations of the model}

The flux of atoms is modelled, using an opposite but equal flux of vacancies $J_{v}$, supposing that atoms diffuse via a vacancy $\left(c_{v}\right.$ is the vacancy concentration) mechanism:

$$
J_{V}=-D_{V} \nabla c_{V}+\frac{D_{V} c_{V}}{k T} Z_{V}^{*} e E
$$

The diffusivity $D_{v}$ of vacancies is

$$
D_{\nu}=D_{o} \exp \left(\frac{-E_{a}}{k T}\right)
$$

where the activation energy $E_{\mathrm{a}}$ has different values in bulk and at grain boundaries $E$ is the electric field and $Z_{\mathrm{Y}}{ }^{*}$ is the effective valence. The conservation law for vacancies

$$
\frac{\partial c_{V}}{\partial t}+\nabla J_{V}=G
$$

introduces a sink/source term $G$ that allows for the creation or annihilation of vacancies at sites as grainboundaries, dislocation lines, surfaces.

$$
G=\frac{c_{v}-c_{v_{e}}}{\tau_{s}}
$$

Where $\tau_{\mathrm{s}}$ is the vacancy lifetime and the number of vacancies in equilibrium with the stress is :

$$
c_{V_{e}}=c_{o} \exp \left(\frac{\sigma \Omega}{k T}\right),
$$

where $c_{0}$ is the vacancy concentration in the absence of any stress effects, $\sigma$ the mechanical stress, $\Omega$ the atomic volume. The creation/ annihilation of vacancies at places as dislocation lines or grain boundaries, cause the dislocation to climb upwards (when vacancies are annihilated) or downwards (when vacancies are created). The climbing motion of an edge dislocation either inserts or removes a vertical plane of atoms in the metal lattice, changing the local crystal volume. We are supposing that vacancies are not created or annihilated at the metal surface, the metal line is considered encapsulated in a strong dielectric. The change in the vacancy concentration from the equilibrium value can be related to the mechanical stress through Hooke's law:

$$
\frac{\partial \sigma}{\partial t}=B \Omega \frac{c_{V}-c_{V_{e}}}{\tau_{s}}
$$

The resistivity of the line is also, related to the change in the vacancy concentration:

$$
\rho=\rho_{o}\left[1+\xi\left(c_{V}-c_{o}\right)\right] .
$$

where

$$
\xi=\frac{1}{N_{a}-c_{V}},
$$

and $\mathrm{N}_{\mathrm{a}}$ is the atomic concentration.

\section{Simulation results}

Simulated structures consist on a metallic stripe with a single polycrystalline region and on a metalic stripe with a near-bamboo microstructure as indicated in fig.l. The metallisation lines have been stressed with a current density of $1 \mathrm{MA} / \mathrm{cm}^{2}$, at $\mathrm{T}=200^{\circ} \mathrm{C}$. The activation energy for vacancies diffusion is $E_{a b u l k}=1.1 \mathrm{eV}$ in bulk and $E_{a g b}=0.6$ $\mathrm{eV}$ for grain boundary. Other relevant parameters in the model assuming an aluminum line: $B=50 \mathrm{GPa}, Z_{\mathrm{v}}{ }^{*}=20$, 
$\tau_{\mathrm{s}}=20 \mathrm{~ms}, \rho_{0}=5 \mu \Omega \mathrm{cm}$. Steady state distribution of mechanical stress for a structure with a single polycrystalline region (region $L$ in $f i g .1$ ) is indicated in fig. 2. The corresponding vacancy distribution is indicated in $\mathrm{fig} .3$.

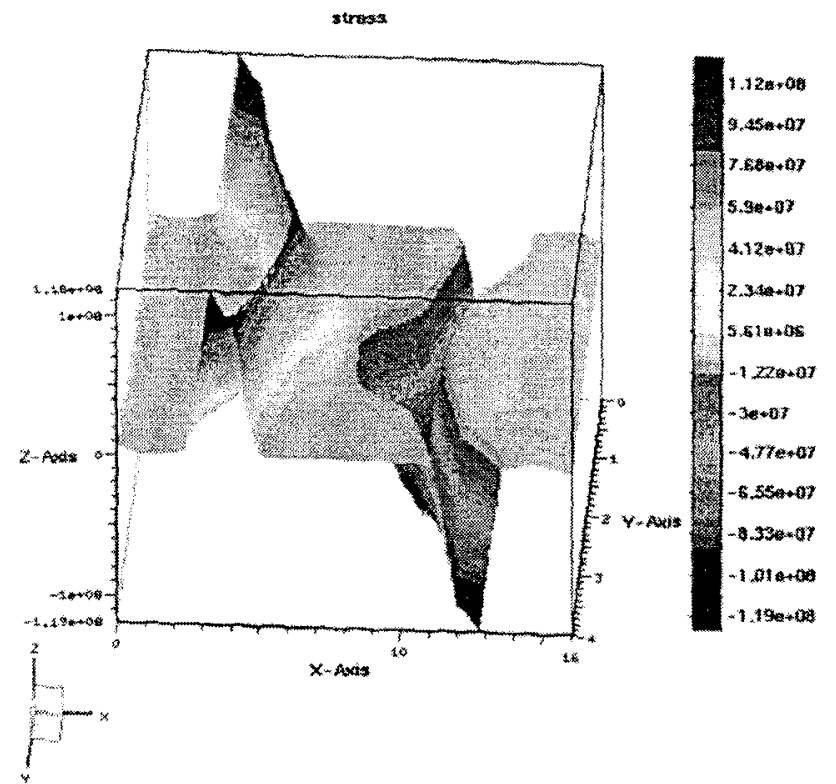

Fig. 2 Mechanical stress distribution for a single polycrystalline region [in $\mathrm{Pa}$ ] after 100 hours of current stress

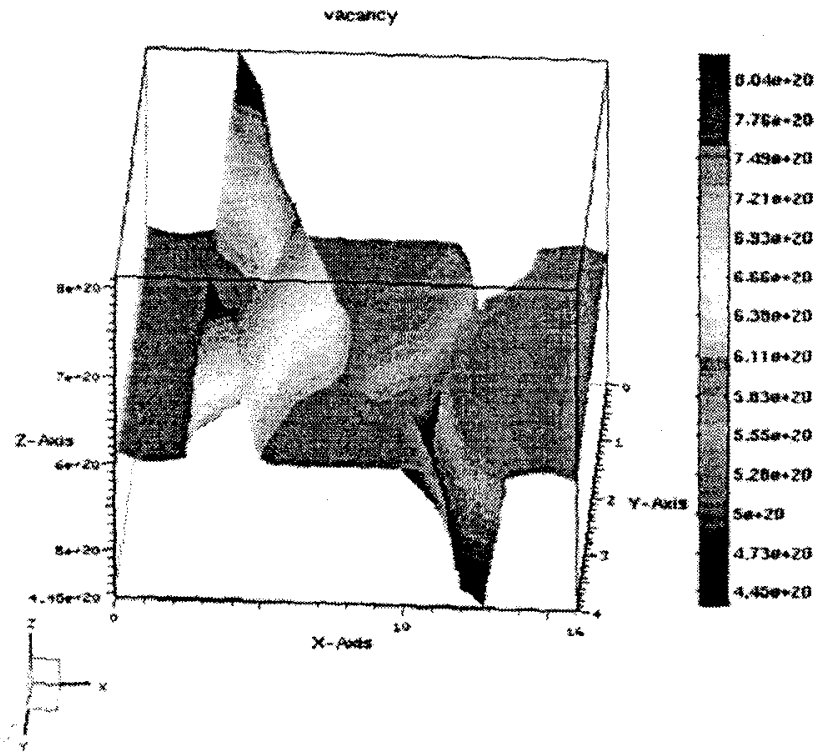

Fig.3 Vacancy distribution for a single polycrystalline region [in $\mathrm{cm}^{-3}$ ] after 100 hours of current stress
Stress distribution for a near-bamboo interconnect line, with the miscrostructure as indicated in fig. 1 is shown in fig.4. The length ( $L$ ) of a polycrystalline region (or cluster region) is $\mathrm{L}=30 \mathrm{\mu m}$ and the distance (d) between the two clusters region is $d=5 \mu \mathrm{m}$. Stress evolution in time and relative resistance change after 1000 hours of current stress are shown in fig. 5 respectively fig. 6 .

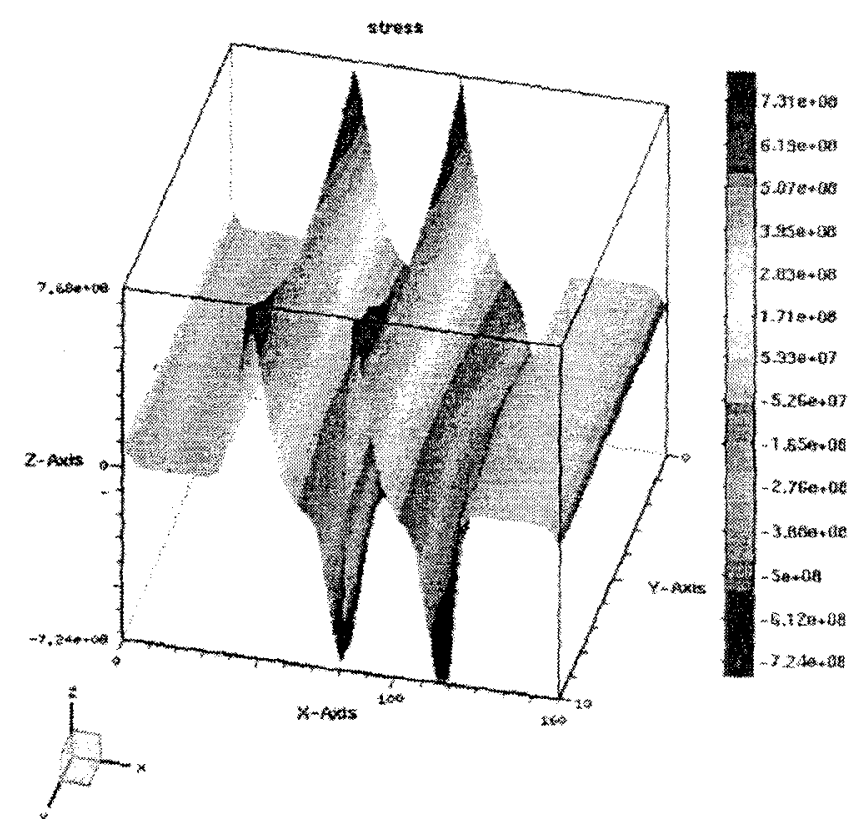

Fig.4 Mechanical stress distribution for a near-bamboo interconnect line [in $\mathrm{Pa}$ ].

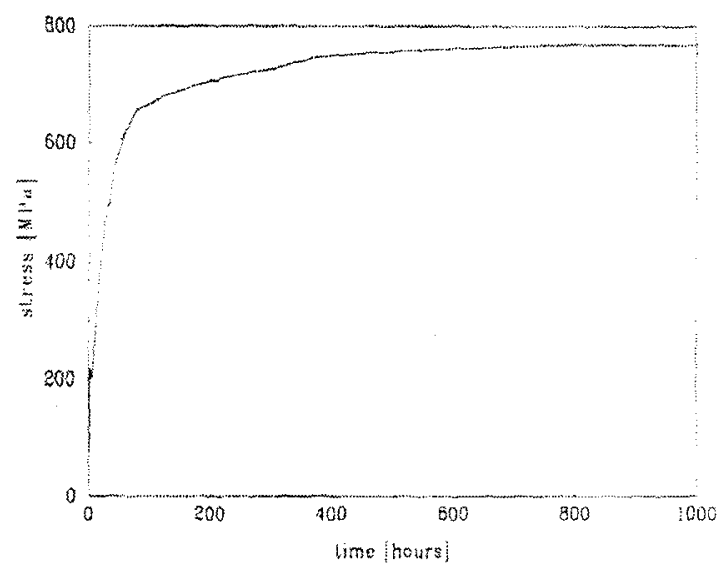

Fig.5 Mechanical stress evolution after 1000 hours of current stress for a near-bamboo interconnect line. 


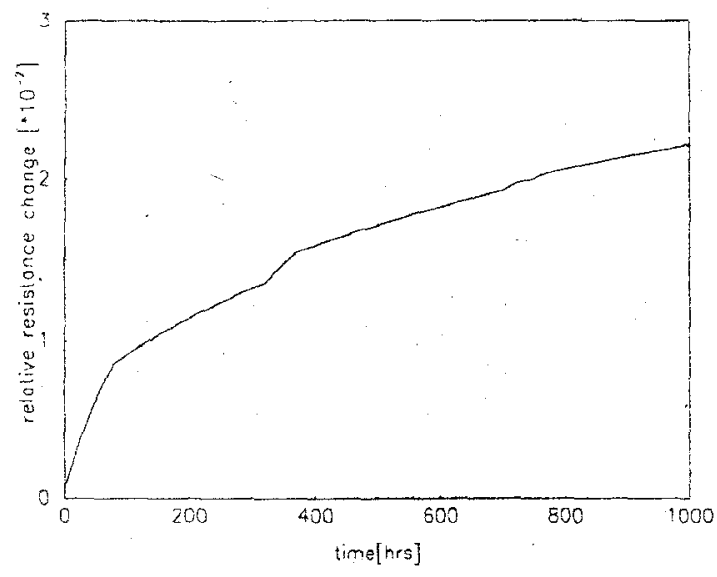

Fig. 6 Relative resistance change in time for a nearbamboo interconnect line.

The cluster length ( $\mathrm{L}$ ) and distance between two cluster regions are important factors on the behaviour of the stress distribution along the interconnect line. Simulations have been performed on a interconnect line with a near-bamboo microstructure, with $\mathrm{L}=20 \mu \mathrm{m}$ (fig. 1) for two different values of the distance between the polycrystalline/cluster regions. For a distance $d=2 * L$ simulation results show that clusters act independently as isolated clusters. If the distance between the two clusters regions is decreased $(\mathrm{d}=\mathrm{L} / 2)$ the stress profiles overlap resulting a strong interaction between the two polycrystalline regions. The differences in behaviour of early resistance change and mechanical stress for isolated clusters and clusters that interact are shown in fig. 7 respectively fig. 8 . The high values of mechanical stress and early resistance change obtained for clusters interaction show the importance of this effect on the reliability of the interconnect line.

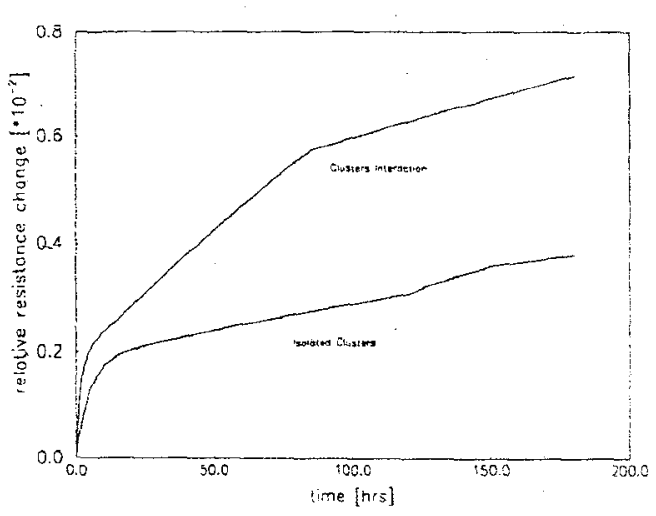

Fig. 7 Relative resistance change in time

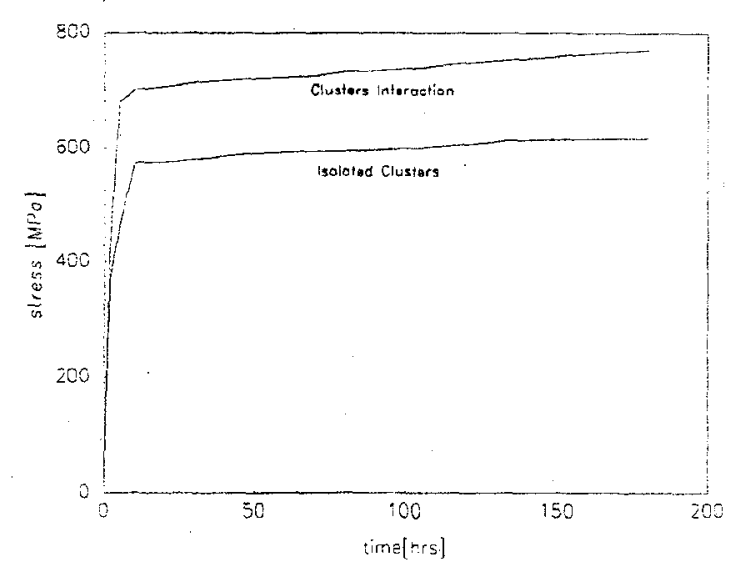

Fig. 8 Mechanical stress distribution

\section{Conclusion}

Important phenomena as clusters interaction, linear increase of resistance change in the early stages of electromigration, can be described by the proposed model, simulation results showing the role of a two dimensional simulator compared to a 1D simulator [6].

\section{References}

[1] M. Shatzkes and J.R. Lloyd, J. Appl. Phys. 59, 3890 (1986)

[2] R. Kirchheim, Acta Mettall. Mater. 40, 309 (1992).

[3] M. A. Korhonen, P. Borgesen, K. N. Tu, and C.-Y. Li, J. Appl. Phys. 73, 3790 (1993).

[4] J. R. Lloyd, ESSDERC Proc., 839, 1996.

[5] J. Niehof, P. A. Flinn, T. J. Maloney, QRE Int. 9, 295, 1993.

[6] B. D. Knowlton, J. J. Clement, R. I. Frank and C. V. Thompson, MRSS Proc. 391, 1995. 